\title{
AUTOMATIC CONTROL SYSTEM OF THE ECCENTRICITY OF CENTER OF GRAVITY FOR OPTIMUM TURNING PERFORMANCE OF A TRACKED VEHICLE ON SOFT TERRAIN
}

\author{
D.T.Tran and T.Muro \\ Faculty of Engineering, Ehime University, 3 Bunkyo-cho, Matsuyama, Japan
}

\begin{abstract}
The objective of the research is to develop an automatic control system of eccentricity of center of gravity of a bulldozer to achieve optimum running performance on soft terrain. For this purpose, a simulation analysis, which can predict turning characteristics of tracked vehicles on soft terrain with high accuracy in a short time, was developed. A measured or desired traction force, desired turning radius etc. can be inputted to the simulation analysis at the site to compute a combination of eccentricity of center of gravity of vehicle and steering ratio at which optimum turning performance can be achieved. The optimum turning performance criteria can be the minimum effective input energy, or minimum amount of sinkage or maximum effective tractive energy etc. In this report, the fist step of the research, i.e. the development of the simulation analysis and its analytical results were reported. The results shows that eccentricity of center of gravity of vehicle has considerable effect on turning performance of tracked vehicle and its value can be set to achieve desired optimum criteria. Furthermore, it is concluded that a substantial reduction of effective input energy can be achieved merely with the adjustment of eccentricity of center of gravity of vehicle.
\end{abstract}

Keywords: eccentricity of center of gravity, optimum criteria, turning performance, tracked vehicle, soft terrain.

\section{INTRODUCTION}

Recent years have witnessed substantial increase of computing speed and considerable decrease of prices of modern computers. With this trend, it will not be far from the time when it is feasible to simulate at real time a lot of working processes so that these processes can be automatically controlled to achieve desired optimum criteria. Similar to other working process, the working processes in contruction work can gain many benefits from the advancement of computer technology. Needless to say, however, that hardware alone cannot help but software must also be developed accordingly to take full benefit of their high computing powers. The research aims at the development of controlling software to be used in the automatic control system of eccentricity of center of gravity of a bulldozer to achieve desired optimum running performance on soft terrain with the use of computers.

As have been known, efficiency of construction machine working on super weak terrain such as muddy condition or fresh concrete is low due to the excessive sinkage of the vehicle and some time excessive inclination in lateral and longitudinal direction when the vehicle is on turning motion.
Therefore, among the desired optimum criteria, minimum input energy and minimum amount of sinkage can be regarded as the most important criteria and the controlling software and controlling system need to be developed with it in mind in the first place. Moreover, the software, i.e. the numerical analysis to predict turning characteristics of tracked vehicle, must be so that it is both fast enough for a real time simulation and accurate enough to efficiently control working process. The numerical analysis, therefore, is not only developed but also verified with series of experiment [1], [2]. In this report, the mechanism of the control system to achieve desired optimum criteria for turning motion and analytical results by a numerical analysis are reported. The result shows that the control of the eccentricity of center of gravity proved to be a very effective method to reduce energy consumption and maximum amount of sinkage. For example, for the case of the investigation in this paper, for the range of effective tractive effort from zero to its maximum value, the effective input energy can be reduced by $20 \%$ in average merely with the moving of the eccentricity of center of gravity of the vehicle within the feasible range of eccentricity adjustment toward the front part of the vehicle. 




Fig.1 Vehicle automatic control system

\section{RESEARCH METHOD}

\subsection{Automatic control system:}

Fig. 1 shows vehicle automatic control system. The vehicle is equipped with a computer, software, control unit of eccentricity of center of gravity, control unit of steering ratio and turning characteristics measuring devices. The system is somewhat similar to the control system developed for straight motion [3]. The desired optimum criteria, desired or to-be-maintained turning characteristics measured at real time are inputted to the simulation analysis. The combination of the eccentricity of center of gravity and steering ratio for desired optimum criteria is then computed at real time. The computed combination is then set by the eccentricity and steering ratio control unit and the vehicle can be run with this set of combination. It is known that, however, the best simulation might not produce the desired accuracy and it is especially true for a real time simulation, which must compute turning characteristics at shortest time possible. Therefore, the optimum criteria might not be fulfilled for the fist run. The inaccuracy of the predicted characteristics can be considered due to an inaccuracy in obtaining terrain-track system constants and in simulation itself. To substitute for the inaccuracy, calibration system is needed. Some of the running characteristics that can be directly measured with ease and accuracy such as effective tractive effort, turning radius, various amount of sinkage, effective driving force, effective input energy etc are measured at real time. These measured characteristics are then fed back to the computer for the purpose of calibration of the software. Some of these characteristics can also be used as input to the numerical analysis as the running characteristics to be maintained (such as a traction force that does not need to be changed).

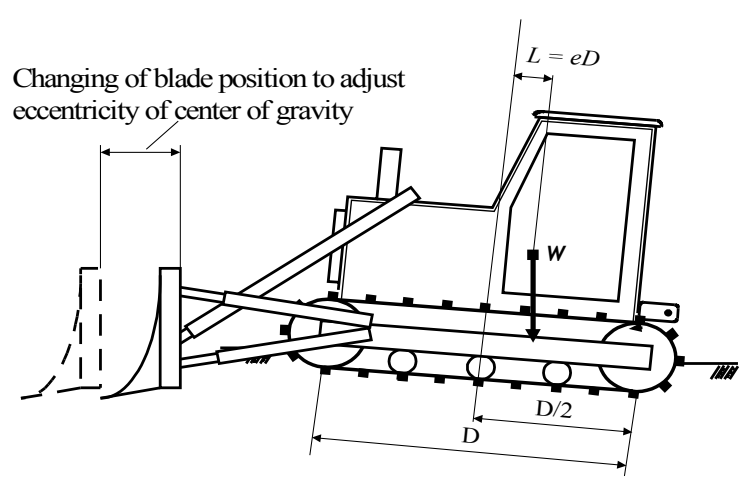

Fig. 2 Adjustment of eccentricity of center of gravity by changing position of blade

\subsection{Control system of eccentricity of center of gravity of actual bulldozers}

The control of eccentricity of center of gravity of the real vehicle can be a problem because of larger mass of the operating vehicle. This part presents the feasibility study on the control of the eccentricity of center of gravity of actual dozers to verify the feasible range within which the eccentricity of center of gravity can be adjusted without adding extra mass. The eccentricity of center of gravity is defined as:

$$
e=\frac{L}{D}
$$

where $L$ is the distance from the geometrical center of the vehicle to the eccentricity of center of gravity. $D$ is the track contact length as shown in the figure 2 . The distance $L$, and so as eccentricity of center of gravity, is regarded as having positive value when the eccentricity of center of gravity is located between geometrical center of the vehicle and tip end of the vehicle. In contrary, it is regarded as having minus value if the eccentricity of center of gravity is located between geometrical center of the vehicle and tip of the front part of the vehicle.

Controlling the eccentricity of center of gravity of the real bulldozer can be executed via the relocation of the controlling mass. The concern is to reduce the operating mass as much as possible so that the sinkage of the vehicle on soft terrain can be reduced. In order not to increase the weight of the vehicle, the mass of the engine, the mass of the hydraulic oil tank together with the hydraulic oil it contains, mass of fuel tanks together with fuel, the mass of bulldozing blade, its operating hydraulic cylinders and supporting beam can be used as controlling mass. A convenient method to adjust the eccentricity of center of gravity is to change the position of blade as shown in figure 2. A blade supporting beams are not an ordinary beams but are the hydraulic cylinders so that the position of blade can be adjusted easily. By our estimate, it is feasible to adjust the eccentricity of center of gravity even within 0.2 ranges without using extra mass. 

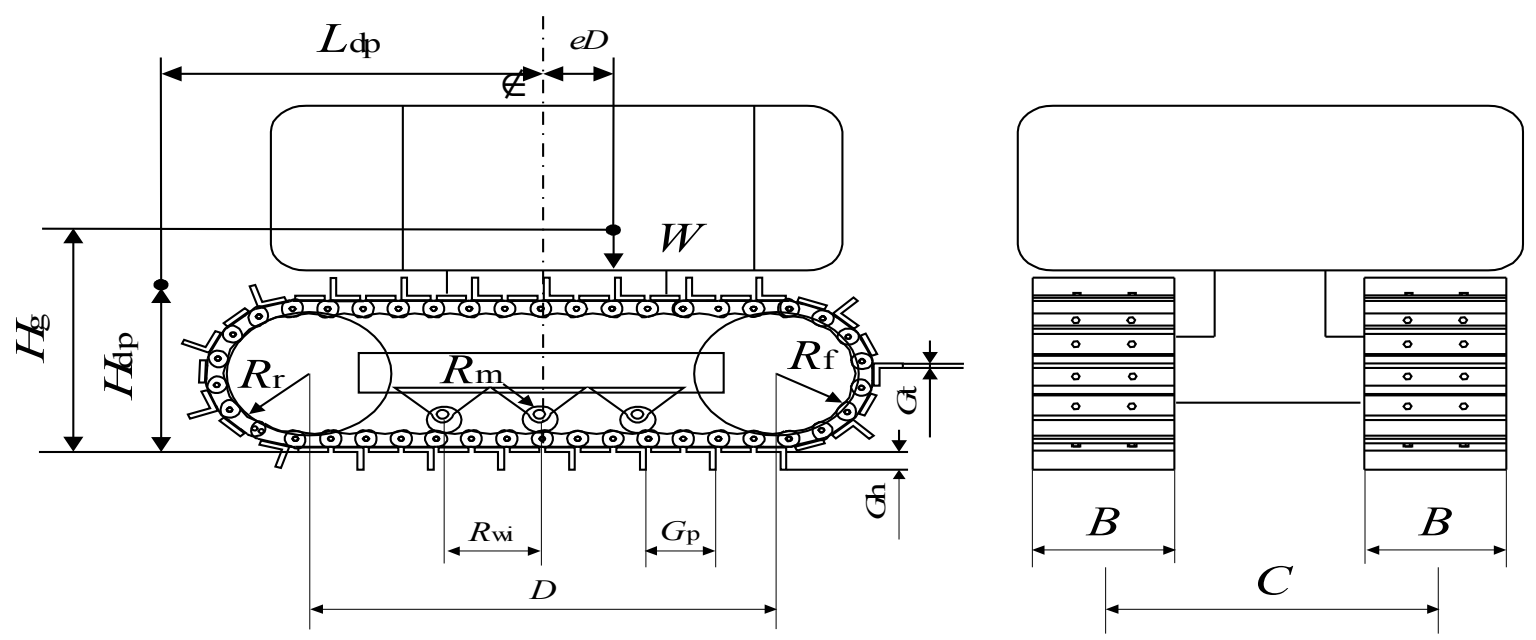

Fig. 3 Outline of model vehicle

Fig. 3 Outline of model vehicle

Table 1 Dimensions of model vehicle

\begin{tabular}{|l|r|l|r|}
\hline Vehicle weight $W(\mathrm{~N})$ & 582 & Grouser height $H(\mathrm{~cm})$ & 1.7 \\
\hline Height of hitch point $H_{\mathrm{dp}}(\mathrm{cm})$ & 15 & Grouser pitch $G_{\mathrm{p}}(\mathrm{cm})$ & 2.55 \\
\hline Dist. geometrical cent.-hitch point $L_{\mathrm{dp}}(\mathrm{cm})$ & 26 & Grouser thickness $G_{\mathrm{t}}(\mathrm{cm})$ & 0.3 \\
\hline Initial track tension $H_{\mathrm{o}}(\mathrm{N})$ & 98.1 & Maximum track deflection $(\mathrm{cm})$ & 0.2 \\
\hline Height of center of gravity $H_{\mathrm{g}}(\mathrm{cm})$ & 15 & Radius of front idler $R_{\mathrm{f}}(\mathrm{cm})$ & 5.4 \\
\hline Contact length $D(\mathrm{~cm})($ changing $)$ & 33 & Radius of rear sprocket $R_{\mathrm{r}}(\mathrm{cm})$ & 5.4 \\
\hline Track width $B(\mathrm{~cm})$ & 10 & Radius of road rollers $R_{\mathrm{m}}(\mathrm{cm})$ & 1.9 \\
\hline Track gauge $C(\mathrm{~cm})$ & 23 & Number of road rollers & 3 \\
\hline Eccentricity of center of gravity & $-0.050 \sim 0.050$ & Road roller interval $R_{\mathrm{wi}}(\mathrm{cm})$ & 6 \\
\hline
\end{tabular}

Table 2. Terrain-track system constants

\begin{tabular}{|c|c|c|c|}
\hline \multicolumn{2}{|c|}{$k_{1}\left(\mathrm{kN} / \mathrm{m}^{\mathrm{n} l+2}\right)$} & \multicolumn{2}{c|}{$n_{1}$} \\
\hline & 1261 & \multicolumn{2}{c|}{1.259} \\
\hline Lon. & $m_{\mathrm{c}}\left(\mathrm{N} / \mathrm{cm}^{2}\right)$ & $m_{\mathrm{f}}$ & $a(1 / \mathrm{m})$ \\
\hline Lat. & 0 & 0.87 & 60 \\
\hline \multicolumn{2}{|c|}{$k_{2}\left(\mathrm{kN} / \mathrm{cm}^{\mathrm{n} 2+2}\right)$} & 0.45 & 370 \\
\hline \multicolumn{2}{|c|}{500} & \multicolumn{2}{|c|}{0.545} \\
\hline \multicolumn{2}{|c|}{$c_{0}\left(\mathrm{~m}^{1+2 \mathrm{cl}-\mathrm{c} 2} / \mathrm{N}^{\mathrm{cl}}\right)$} & $c_{1}$ & $c_{2}$ \\
\hline \multicolumn{2}{|c|}{4.957} & 0.81 & 0.7 \\
\hline
\end{tabular}




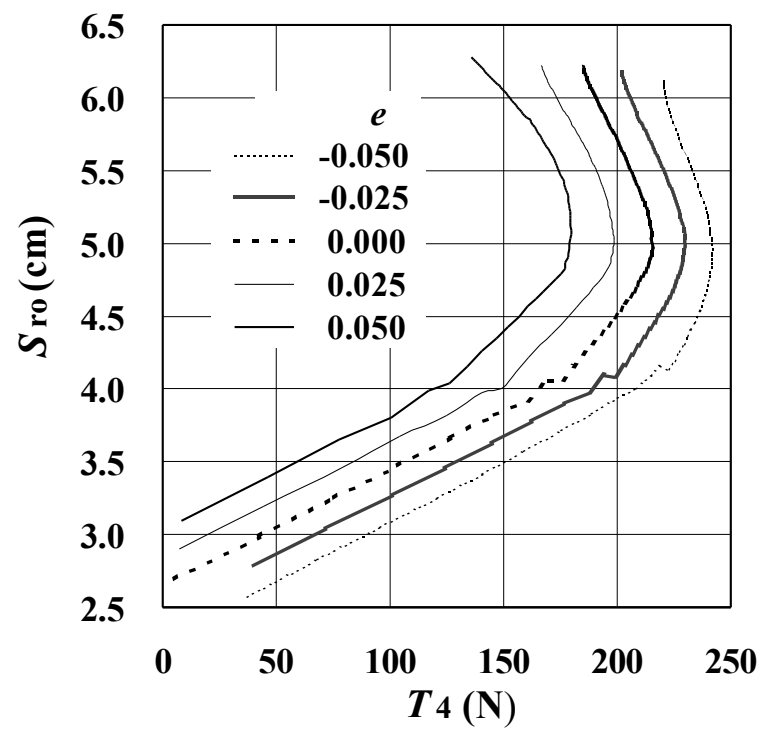

Fig. 5 Influence of eccentricity of center of gravitye on relationship between amount of sinkage of outer rear track $S$ ro and effective tractive effort $T 4$

\subsection{Object of the Research}

The investigation is carried out for a case of the roughly $1 / 10$-scaled model of an actual bulldozer. The outline of the model vehicle and its dimensions, which is used as input to the simulation analysis, is shown in Fig. 3 and Table 1, respectively. The circumferential speed of outer track was fixed 100 $\mathrm{cm} / \mathrm{s}$ and the turning radius was $150 \mathrm{~cm}$.

The soil on which the vehicle was running was airdried sandy soil. The terrain-track system constants for the given track-soil system were determined by loading unloading test and traction test and are presented in Table 2 .

The investigation was carried out for five cases of eccentricity of center of gravity of $-0.050,-0.025$, $0.000,0.025$ and 0.050 . For each case of the eccentricity of center of gravity, the effective traction force was changed from 0 to its maximum values.

\section{RESULT AND DISCUSSIONS}

This chapter presents the result of the investigation on the influence of eccentricity of center of gravity on turning characteristics of a model vehicle running on soft terrain.

Fig. 5 shows the influence of eccentricity of center of gravity $e$ on the relationship between sinkage of rear sprocket of outer track $S_{\text {ro, }}$ i.e. maximum amount of sinkage, and effective tractive effort $T_{4}$. The figure shows the expected result that the sinkage of rear sprocket of outer track decreases with the decrement of the eccentricity of center of gravity. In average, the decrement of eccentricity of center of gravity from

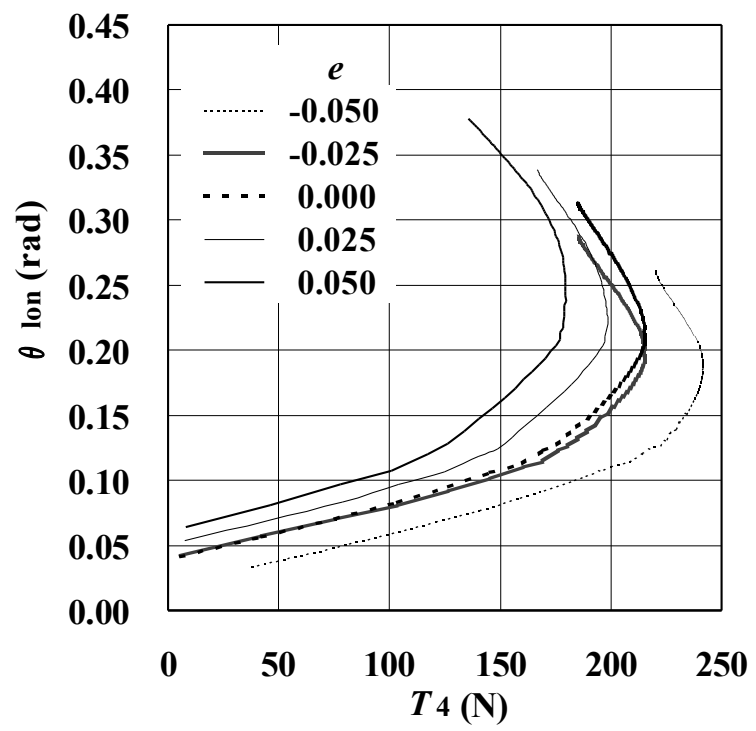

Fig. 6 Influence of eccentricity of center of gravitye on relationship between longitudinal inclination angle $\theta$ lon and effective tractive effort $T 4$

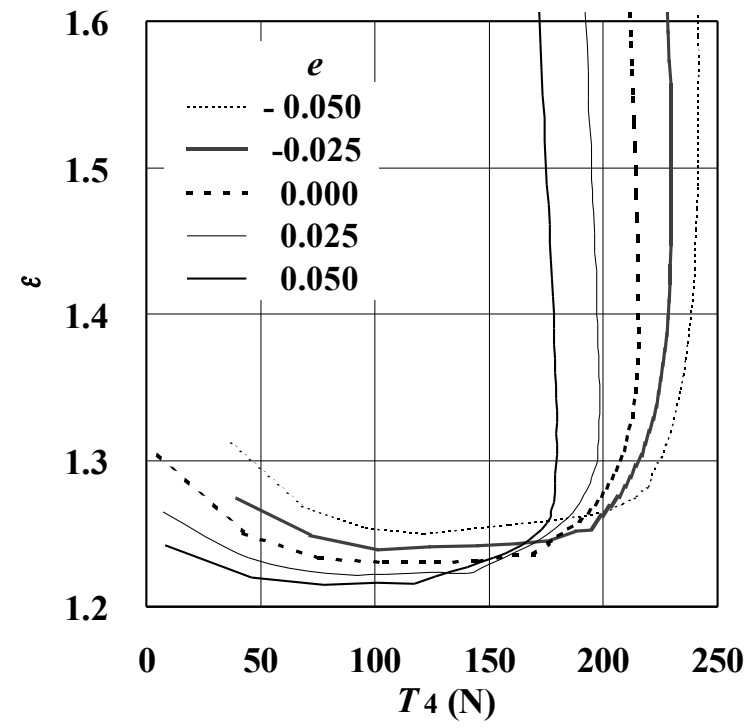

Fig. 7 Influence of eccentricity of center o gravity $e$ on relationship between steering ratio $\varepsilon$ and effective tractive effort $T 4$

0.050 to -0.050 brings about the decrease of about $20 \%$ of amount of sinkage of rear sprocket of outer track.

Fig. 6 shows the influence of eccentricity of center of gravity $e$ on the relationship between longitudinal inclination angle $\theta_{\text {lon }}$ and effective tractive effort $T_{4}$. As expected, the longitudinal inclination angle decreases with the decrement of eccentricity of center of gravity. Due to this phenomenon, the average and maximum amount of sinkage can be reduced which leads to the reduction of effective input energy with the decrease of eccentricity of center of gravity as can be seen in the later presented fig. 12 .

Fig. 7 shows the influence of eccentricity of center 


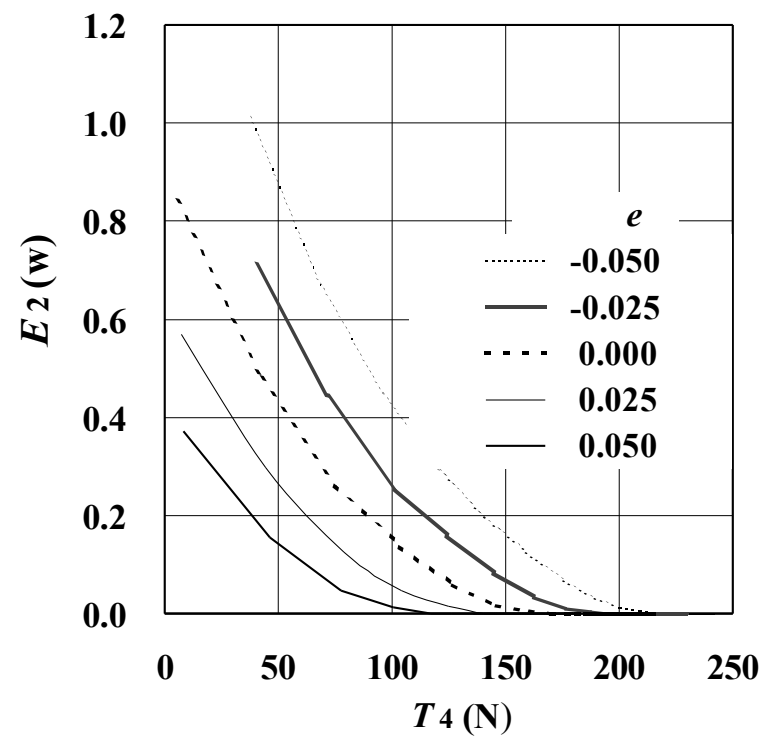

Fig. 8 Influence of eccentricity of center of gravitye on relationship between land lomotion energy $E 2$ and effective tractive effort $T 4$ locomotion energy resistance $E_{2}$ and effective tractive effort $T_{4}$. The land locomotion energy increases with the decrement of eccentricity of center of gravity. The phenomenon can be explained by the fact that with the decrement of eccentricity of center of gravity, i.e. with the moving of the eccentricity of center of gravity toward the front part of the vehicle, the sinkage of front idlers increases. Therefore, the land locomotion, which is computed as function of sinkage of front idlers, increases accordingly.

Fig. 9 shows the influence of eccentricity of center of gravity $e$ on the relationship between slip energy $E_{3}$ and effective tractive effort $T_{4}$. The slip energy is observed to be decreasing with the decrement of the eccentricity of center of gravity, i.e. with the moving the eccentricity of center of gravity toward the front part of the vehicle. The decrease of slip energy with the decrement of eccentricity of center of gravity is due to the decrease of thrust with the decrement of eccentricity of center of gravity. The decrease of thrust with the decrement of eccentricity of center of gravity, in return, is needed to maintain force balance

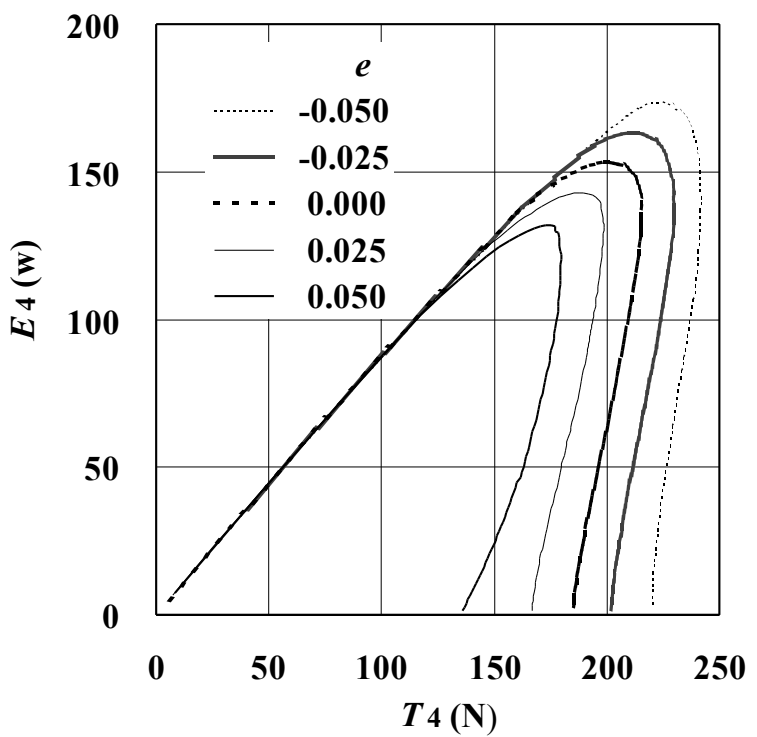

Fig. 10 Influence of eccentricity of center of gravity $e$ on relationship between effective tractivs energy $E 4$ and effective tractive effort $T 4$

of gravity $e$ on the relationship between steering ratio $\varepsilon$ and effective tractive effort $T_{4}$. In general, it is observed from the figure that the steering ratio increases with the decrement of eccentricity of center of gravity. The graph can be used to control turning radius for a given desired effective tractive effort by adjusting the eccentricity of center of gravity or to set the combination of the steering ratio and eccentricity of center of gravity to achieve desired optimum criteria.

Fig. 8 shows the influence of eccentricity of center of gravity $e$ on the relationship between land in longitudinal direction of the vehicle because of the decrease of longitudinal angle with the decrement of eccentricity of center of gravity as can be observed in fig. 6 . 

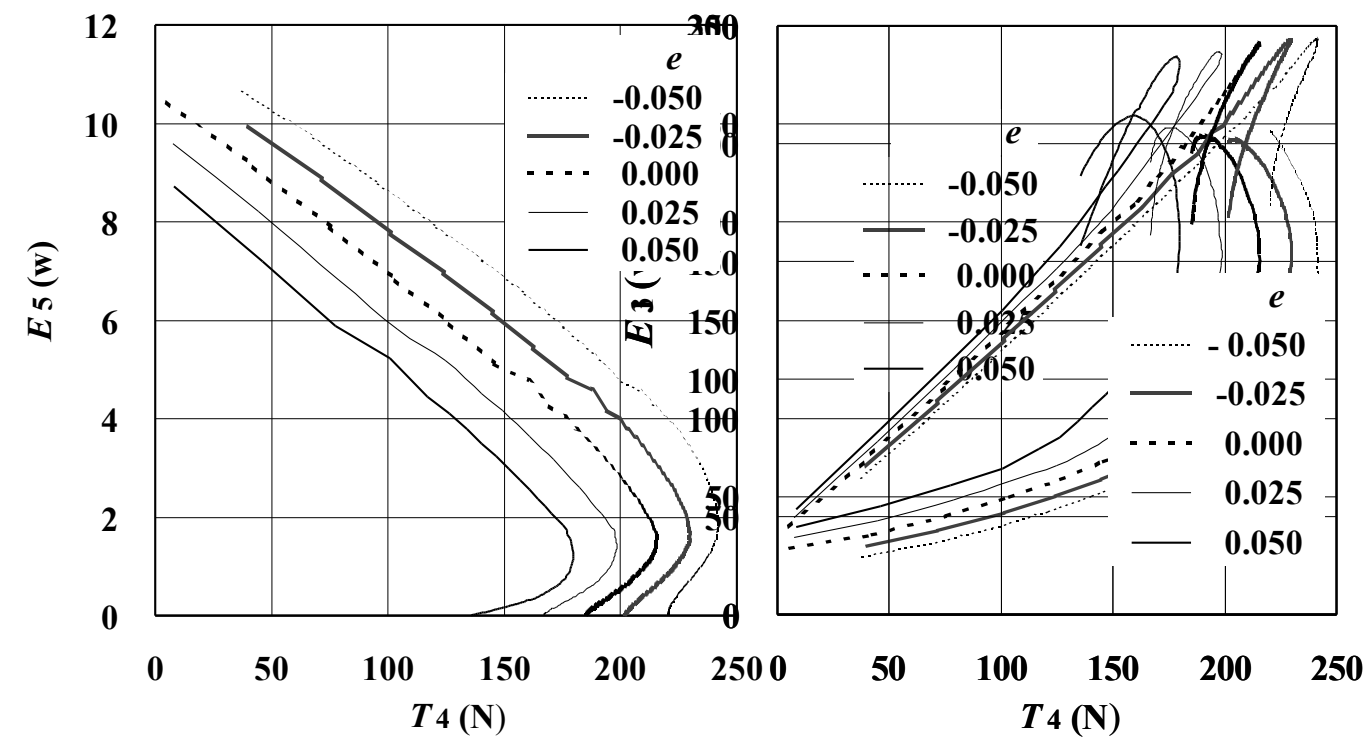

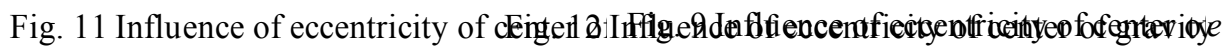

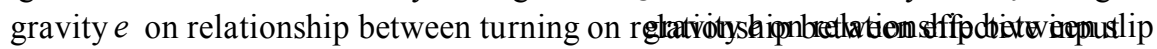

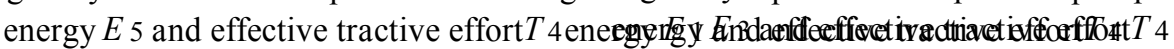

Fig. 10 shows the influence of eccentricity of center of gravity $e$ on the relationship between effective tractive energy $E_{4}$ and effective tractive effort $T_{4}$. It is observed that the eccentricity of center of gravity has virtually no influence on the effective tractive energy for the smaller range of effective tractive effort. For a range of effective tractive efforts near its maximum value, it is observed that the effective tractive effort increases slightly with the decrement of eccentricity of center of gravity.

Fig. 11 shows the influence of eccentricity of center of gravity $e$ on the relationship between turning energy $E_{5}$ and effective tractive effort $T_{4}$. In general, the turning energy increases with the decrement of eccentricity of center of gravity. Although the increase of turning energy with the decrement of eccentricity of center of gravity is rather significant, its ratio to the effective input energy decreases greatly with the increase of effective tractive effort.

Fig. 12 shows the influence of eccentricity of center of gravity $e$ on the relationship between effective input energy $E_{1}$ and effective tractive effort $T_{4}$. The effective input energy decreases with the decrement of eccentricity of center of gravity, i.e. with the movement of eccentricity of gravity center toward front part of the vehicle. The decrement of eccentricity of center of gravity from 0.05 to -0.05 brings about an average reduction of the required effective input energy of about $20 \%$. Moreover, it is observed that the more the effective tractive effort is required, the more energy saving can be achieved. For example, for the case of effective effort of $170 \mathrm{~N}$, the required effective input energy is reduced by $25 \%$ when the eccentricity of center of gravity is reduced from 0.05 to -0.05 . Therefore, it can be concluded that the control of eccentricity of center of gravity is a very effective method to reduce the energy consumption.

\section{CONCLUSIONS}

Automatic control system of the eccentricity of center of gravity to achieve optimum performance using numerical analysis was proposed. The investigation on the effect of the eccentricity of center of gravity on turning characteristics shows that:

(1) Desired optimum criteria can be achieved with the adjustment of eccentricity of center of gravity without having to use extra mass as a mean to control eccentricity of center of gravity.

(2) Considerable reduction of the effective input energy can be achieved with the moving of the eccentricity of center of gravity toward the front part of the vehicle. The decrement of eccentricity of center of gravity from 0.05 to -0.05 brings about an average reduction of the required effective input energy of about $20 \%$.

(3) Decrement of eccentricity of center of gravity improves turning characteristics by reducing maximum amount of sinkage, longitudinal inclination angle, slip energy and effective input energy despite the fact that it increases land locomotion energy and turning energy.

\section{REFERENCES}

[1] D. T. Tran and T. Muro: Numerical analysis to predict turning characteristics of rigid suspension tracked vehicle, Journal of Terramechanics Vol. 36 
No.4, pp.183-196, October 1999.

[2] D. T. Tran, T. Muro, S. Kawahara and M. Hirakawa: Influence of track shape ratio on turning characteristics of a tracked vehicle running on fresh concrete, Journal of Construction Management and Engineering, JSCE, No. 651/VI-47, pp. 169-180, June 2000.

[3] T.Muro, T. Shigematsu: Optimal design method of a wheel travel system for special purpose and robotic vehicle operating over sloped weak sandy terrain, Proc. of the 14th Int. Symposium on Automation and Robotics in Construction, Pittsburgh, USA, pp.312-319, June 1997. 